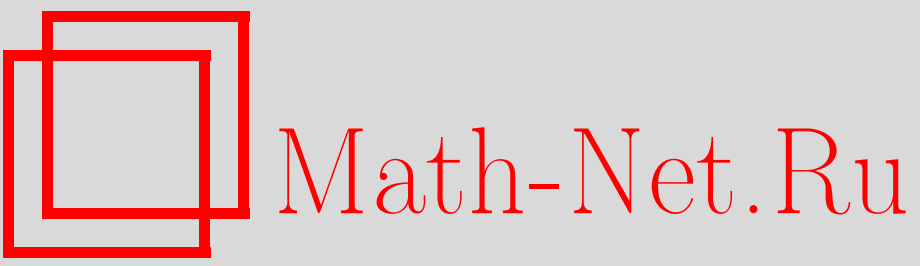

Л. В. Жуковская, А. М. Савченко, М. Б. Садовникова, Низкочастотная спиновая ветвь колебаний в системах с обменным взаимодействием, ТМФ, 2004, том 138, номер 1, 139-143

DOI: https://doi.org/10.4213/tmf15

Использование Общероссийского математического портала Math-Net.Ru подразумевает, что вы прочитали и согласны с пользовательским соглашением

http: //www . mathnet.ru/rus/agreement

Параметры загрузки:

IP : 18.234 .156 .22

26 апреля 2023 г., 14:41:07 
ТЕОРЕТИЧЕСКАЯ

И МАТЕМАТИЧЕСКАЯ

ФИЗИКА

Том 138, № 1

январь, 2004

(С 2004 г. Л.В. Жуковская*, А. М. Савченко*, М.Б. Садовникова*

\section{НИЗКОЧАСТОТНАЯ СПИНОВАЯ ВЕТВЬ КОЛЕБАНИЙ В СИСТЕМАХ С ОБМЕННЫМ ВЗАИМОДЕЙСТВИЕМ}

\footnotetext{
Определена спиновая ветвь колебаний с частотой $\omega=(\mu H / \hbar)\left(k / k_{\mathrm{c}}\right)^{2}$ в парамагнитной фазе для систем с обменным взаимодействием при наличии внешнего магнитного поля $\vec{H}$. Показано, что спиновая составляющая тока в линейном режиме возбуждения спиновых поперечных мод имеет резонансный характер.
}

Ключевые слова: спиновые флуктуации, спиновая динамика, обменное взаимодействие, ток, тензор динамической магнитной восприимчивости.

Обменное взаимодействие играет важную роль в коллективных электронных взаимодействиях [1]. Оно приводит к возникновению в спектре спиновых возбуждений колебательных мод, которые оказываются реальными в области значений волнового вектора $k>k_{\mathrm{c}}=2 \pi /\left\langle r_{\mathrm{c}}\right\rangle$, где $\left\langle r_{\mathrm{c}}\right\rangle$ - обменный радиус коррелящии.

В области же малых волновых векторов, т.е. при $k<k_{\mathrm{c}}$, без внешнего магнитного поля спектр спиновых флуктуаций является диффузионным вследствие отсутствия в системе электронных спинов дальнего магнитного порядка [2]. Представляет определенный интерес исследование области малых $k$ при наличии внешнего магнитного поля, чему и посвящена данная работа.

Будем исходить из того, что гамильтониан спиновой системы имеет вид ([3]; детальное получение см. [2], с. 134)

$$
H_{\mathrm{S}}=\int d \vec{x}\left[\frac{m^{2}}{2 \chi}+\frac{1}{2 k_{\mathrm{c}}^{2}} J_{0} s A_{\nu}^{2}-\frac{1}{2} J_{0} \Omega^{2}-\mu(\vec{H}+\vec{h}, \vec{m}+\vec{\Omega})\right] .
$$

Здесь $\vec{\Omega}$ - намагниченность, соответствующая парамагнитной спиновой степени свободы ("поле" спиновой системы); $\vec{m}$ - парамагнитный момент, неравновесная компонента которого определяется производной намагниченности по времени (импульс спиновой системы); $\vec{A}_{\nu}=\partial \vec{\Omega} / \partial x_{\nu}, \quad \nu=1,2,3 ; \vec{H}=\vec{z} H, \vec{h}$ - постоянное и переменное внешние магнитные поля; $J_{0}=\int d \vec{x} J(\vec{x})$ - потенциал антиферромагнитного обменного взаимодействия; $s=1 / 2$ - спин электрона; $\chi=1 /\left(J_{0} s\right)$ - эффективная парамагнитная восприимчивость (эффективная масса спиновой системы); $k_{\mathrm{c}}=2 \pi /\left\langle r_{\mathrm{c}}\right\rangle ;\left\langle r_{\mathrm{c}}\right\rangle^{2}=$ $\int d \vec{x} x^{2} J(\vec{x}) / \int d \vec{x} J(\vec{x})$ - обменный радиус корреляции в системе электронных спинов; $\mu=g \mu_{b}, g=2, \mu_{b}$ - магнетон Бора.

\footnotetext{
* Московский государственный университет, Москва, Россия. E-mail: 1.joukovskaya@mtu-net.ru
} 
Используя метод скобок Пуассона [4], можно записать уравнения движения для векторов $\vec{m}, \vec{A}_{\nu}, \vec{\Omega}$. В общем случае они имеют вид

$$
\dot{\vec{m}}=\left\{H_{s}, \vec{m}\right\}, \quad \dot{\vec{A}}_{\nu}=\left\{H_{s}, \vec{A}_{\nu}\right\}, \quad \dot{\vec{\Omega}}=\left\{H_{s}, \vec{\Omega}\right\} .
$$

Чтобы записать эти уравнения в явном виде, необходимо вычислить скобки Пуассона для векторов $\vec{m}, \overrightarrow{A_{\nu}}, \vec{\Omega}$. Скобки, отличные от нуля, имеют вид

$$
\begin{gathered}
\left\{m^{\alpha}, \Omega^{\beta}\right\}=\delta^{\alpha \beta}, \quad\left\{\Omega^{\alpha}, m^{\beta}\right\}_{a}=\delta^{\alpha \beta}, \\
\left\{m^{\alpha}, m^{\beta}\right\}=e^{\alpha \beta \gamma} m^{\gamma}, \quad\left\{m^{\alpha}, m^{\beta}\right\}_{a}=\delta^{\alpha \beta}, \\
\left\{m^{\alpha}, A_{\nu}^{\beta}\right\}=\delta^{\alpha \beta} \nabla_{\nu}-e^{\alpha \beta \gamma} A_{\nu}^{\gamma}, \quad\left\{m^{\alpha}, A_{\nu}^{\beta}\right\}_{a}=2 m^{\alpha} A_{\nu}^{\beta}, \\
\left\{\Omega^{\alpha}, \Omega^{\beta}\right\}=e^{\alpha \beta \gamma} \Omega^{\gamma}, \quad\left\{\Omega^{\alpha}, \Omega^{\beta}\right\}_{a}=\delta^{\alpha \beta}, \quad \alpha, \beta, \gamma=1,2,3 .
\end{gathered}
$$

Скобки с индексом “ $a$ ” представляют собой антикоммутируюшие скобки Пуассона.

Запишем уравнения движения для векторов $\vec{m}, \overrightarrow{A_{\nu}}, \vec{\Omega}$ :

$$
\begin{gathered}
\dot{\vec{m}}=\frac{1}{k_{\mathrm{c}}^{2}} J_{0} s \nabla_{\nu} \vec{A}_{\nu}+J_{0} s \vec{\Omega}+\mu[\vec{H}+\vec{h}, \vec{m}], \\
\dot{\vec{A}}_{\nu}=\frac{1}{\chi}\left\{\nabla_{\nu}(\vec{m}-\chi \mu(\vec{H}+\vec{h}))-\left[\vec{A}_{\nu},(\vec{m}-\chi \mu(\vec{H}+\vec{h}))\right]\right\}, \\
\dot{\vec{\Omega}}=\frac{1}{\chi} \vec{m}-\mu(\vec{H}+\vec{h})+\mu[\vec{H}+\vec{h}, \vec{\Omega}] .
\end{gathered}
$$

Линеаризуя полученные уравнения с помошью формул $\vec{A}_{\nu}=z \vec{A}_{\nu 0}+\delta \overrightarrow{A_{\nu}}, \vec{m}=\chi \mu \vec{H}+$ $\delta \vec{m}, \vec{\Omega}=-\chi \mu \vec{H}+\delta \vec{\Omega}$, в случае слабого постоянного $\vec{H}$ и переменного $\vec{h}=\left(h^{x}, h^{y}\right)$ внешних магнитных полей можно записать для них соответствуюшие выражения в алгебраической форме:

$$
\begin{gathered}
-i \omega \delta m^{x}=\frac{1}{k_{\mathrm{c}}^{2}} J_{0} s i k_{\nu} \delta A_{\nu}^{x}+J_{0} s \delta \Omega^{x}-\mu H \delta m^{y}+\chi \mu H \mu h^{y}+\mu h^{x} \\
-i \omega \delta m^{y} x=\frac{1}{k_{\mathrm{c}}^{2}} J_{0} s i k_{\nu} \delta A_{\nu}^{y}+J_{0} s \delta \Omega^{y}+\mu H \delta m^{x}-\chi \mu H \mu h^{x} y+\mu h^{y} \\
-i \omega \delta A_{\nu}^{x}=\frac{1}{\chi} i k_{\nu}\left(\delta m^{x}-\chi \mu h^{x}\right) \\
-i \omega \delta A_{\nu}^{y}=\frac{1}{\chi} i k_{\nu}\left(\delta m^{y}-\chi \mu h^{y}\right) \\
-i \omega \delta \Omega^{x}=\frac{1}{\chi} \delta m^{x}-\mu h^{x}-\mu H \delta \Omega^{y}+\mu h^{y} \Omega_{0} \\
-i \omega \delta \Omega^{y}=\frac{1}{\chi} \delta m^{y}-\mu h^{y}+\mu H \delta \Omega^{x} y+\mu h^{x} \Omega_{0} \\
\Omega_{0}=-\chi \mu H, A_{\nu 0} \rightarrow 0 .
\end{gathered}
$$

Вводя безразмерные величины $z=\omega /\left(J_{0} s\right), z_{0}=\mu H /\left(J_{0} s\right), z^{\alpha}=\mu h^{\alpha} /\left(J_{0} s\right), k_{\nu} / k_{\mathrm{c}}=$ $q_{\nu}$ и производя соответствующие упрошения, получаем следуюшую систему уравнений:

$$
\begin{gathered}
-z^{2} \delta m^{x}=-q^{2} \delta m^{x}+i z z_{0} \delta m^{y}-i z \delta \Omega^{x}+\left(q^{2}-i z\right) z^{x}-i z z_{0} z^{y}, \\
-z^{2} \delta m^{y}=-q^{2} \delta m^{y}-i z z_{0} \delta m^{x}-i z \delta \Omega^{y}+\left(q^{2}-i z\right) z^{y}+i z z_{0} z^{x}, \\
-i z \delta \Omega^{x}=-z_{0} \delta \Omega^{y}+\delta m^{x}-z^{x}-z_{0} z^{y} \\
-i z \delta \Omega^{y}=-z_{0} \delta \Omega^{x}+\delta m^{y}-z^{y}+z_{0} z^{x} .
\end{gathered}
$$


Решение уравнений (8) позволяет определить полную неравновесную намагниченность $\Delta M^{x, y}=\delta m^{x, y}+\delta \Omega^{x, y}$. Ее компоненты имеют вид

$$
\begin{aligned}
& \Delta M^{x}=-\frac{1}{\Delta(z, \vec{q})}\left[z^{2} q^{2}\left(z^{2}+z_{0}^{2}+1-q^{2}\right) z^{x}-i z z_{0} q^{2}\left(2 z^{2}-q^{2}\right) z^{y}\right], \\
& \Delta M^{y}=-\frac{1}{\Delta(z, \vec{q})}\left[i z z_{0} q^{2}\left(2 z^{2}-q^{2}\right) z^{x}+z^{2} q^{2}\left(z^{2}+z_{0}^{2}+1-q^{2}\right) z^{y}\right],
\end{aligned}
$$

где

$$
\Delta(z, \vec{q})=\left[z\left(z^{2}+z_{0}^{2}+1-q^{2}\right)-z_{0}\left(2 z^{2}-q^{2}\right)\right]\left[z\left(z^{2}+z_{0}^{2}+1-q^{2}\right)+z_{0}\left(2 z^{2}-q^{2}\right)\right] .
$$

Перейдем к компонентам, соответствующим правой и левой поляризации полной намагниченности:

$$
\begin{gathered}
\Delta M^{ \pm}=\Delta M^{x} \pm i \Delta M^{y} \\
\Delta M^{ \pm}=-\frac{1}{\Delta(z, \vec{q})} z q^{2}\left[z\left(z^{2}+z_{0}^{2}+1-q^{2}\right) \mp z_{0}\left(2 z^{2}-q^{2}\right)\right] z^{ \pm} \\
z^{ \pm}=z^{x} \pm i z^{y}
\end{gathered}
$$

Для сравнения выпишем также продольную компоненту полной неравновесной намагниченности $\Delta M^{z}$ :

$$
\Delta M^{z}=-\frac{q^{2} z^{2}}{z^{2}+1-q^{2}} .
$$

Вычислим теперь компоненты тензора динамической магнитной восприимчивости, которые определяются следующим образом:

$$
\chi^{\alpha, \beta}=\frac{\partial \Delta M^{\alpha}}{\partial z^{\beta}}, \quad \alpha, \beta=+,-, z .
$$

Таким образом, тензор динамической магнитной восприимчивости имеет диагональный вид, и его компоненты равны

$$
\begin{aligned}
& \chi^{++}(z, \vec{q})=-\frac{z q^{2}}{z\left(z^{2}+z_{0}^{2}+1-q^{2}\right)+z_{0}\left(2 z^{2}-q^{2}\right)} \\
& \chi^{--}(z, \vec{q})=-\frac{z q^{2}}{z\left(z^{2}+z_{0}^{2}+1-q^{2}\right)-z_{0}\left(2 z^{2}-q^{2}\right)} \\
& \chi^{z z}(z, \vec{q})=-\frac{q^{2}}{z^{2}+1-q^{2}} .
\end{aligned}
$$

Полюсы тензора динамической магнитной восприимчивости определяют спектр спиновых возбуждений рассматриваемой системы. Отметим, что полюсы продольной компоненты тензора в области малых $q(q<1)$ чисто мнимые, что соответствует диффузионной продольной спиновой моде. Гораздо больший интерес представляют собой полюсы поперечных компонент $\chi^{++}, \chi^{--}$.

Рассмотрим полюсы компоненты $\chi^{++}$. Их можно определить из уравнения

$$
z^{3}+2 z_{0} z^{2}+z\left(z_{0}^{2}+1-q^{2}\right)-z_{0} q^{2}=0,
$$


имеющего один действительный корень, который в области малых $q$ равен

$$
z_{\vec{q}} \cong z_{0} q^{2}
$$

Соответствующая ему частота спиновых неоднородных колебаний имеет вид

$$
\hbar \omega_{\vec{q}} \cong \mu H q^{2} .
$$

Заметим, что данная ветвь спиновых колебаний (спиновых флуктуаций) существует только в отличном от нуля внешнем магнитном поле и по симметрии спектра аналогична геликонам в магнитоактивной плазме [5]. Таким образом, возникает возможность экспериментального наблюдения этой ветви. Однако здесь имеются определенные трудности. Данная мода является низкочастотной в области малых $q$, и поэтому ее нельзя возбудить в линейном режиме переменным магнитным полем резонансным способом. Указанная ветвь спиновых колебаний может быть наблюдаема при нерезонансном возбуждении ее высокочастотным электромагнитным полем. В этом случае она представляет из себя набор сателлитов, расположенных вблизи основной спектральной линии. Полученный результат представляет интерес с точки зрения экспериментального наблюдения найденной ветви спиновых колебаний, в том числе и для высокотемпературных сверхпроводяших систем.

Исследуем далее нелинейное возбуждение спиновых поперечных мод, для чего обратимся к уравнению (6). Линеаризуя уравнение (6) с помощью тех же, что и ранее, формул $\vec{m}=\chi \mu \vec{H}+\delta \vec{m}, \vec{\Omega}=-\chi \mu \vec{H}+\delta \vec{\Omega}$, где $\delta \vec{m}$ и $\delta \vec{\Omega}$ - малые изменения спиновых переменных $\vec{m}$ и $\vec{\Omega}$ в случае слабого постоянного $\vec{H}$ и переменного $\vec{h}=\left(0,0, h^{z}\right)$ внешних магнитных полей, и производя соответствуюшие упрошения, приходим к системе уравнений

$$
\begin{aligned}
& -i z \delta \Omega^{x}=\delta m^{x}-\left(z_{0}+z^{z}\right) \delta \Omega^{y}, \\
& -i z \delta \Omega^{y}=\delta m^{y}+\left(z_{0}+z^{z}\right) \delta \Omega^{x},
\end{aligned}
$$

решая которую, получаем

$$
\begin{aligned}
\delta \Omega^{x} & =\frac{i z \delta m^{x}+\left(z_{0}+z^{z}\right) \delta m^{y}}{z^{2}-\left(z_{0}+z^{z}\right)^{2}}, \\
\delta \Omega^{y} & =\frac{i z \delta m^{y}-\left(z_{0}+z^{z}\right) \delta m^{x}}{z^{2}-\left(z_{0}+z^{z}\right)^{2}} .
\end{aligned}
$$

Таким образом, выше нами было исследовано нелинейное возбуждение спиновых поперечных мод и получены выражения для поперечных компонент намагниченности.

Далее на основе полученных результатов может быть определена величина плотности тока намагниченности, связанная со спиновыми колебаниями [6]:

$$
\vec{j}_{m}=\mu_{b} c n \operatorname{rot} \Delta \vec{M}
$$

где $\mu_{b}$ - магнетон Бора, $n$ - конщентрация носителей в полупроводнике, $c$ - скорость света, $\Delta \vec{M}$ - полная неравновесная намагниченность. Величину $z$-компоненты плотности тока намагниченности (20) можно представить в виде

$$
j_{m z}=\frac{\mu_{b} c n k_{\mathrm{c}}}{2}\left(q^{-} \Delta M^{+}-q^{+} \Delta M^{-}\right),
$$


где

$$
\begin{gathered}
\Delta M^{+}(\vec{q}, z) \cong-\frac{z q^{2} z^{+}}{\left(z-z_{0} q^{2}\right)\left[z^{2}+z z_{0}\left(2+q^{2}\right)+z_{0}^{2}+1-q^{2}\right]}, \\
\Delta M^{-}(\vec{q}, z) \cong-\frac{z q^{2} z^{-}}{\left(z+z_{0} q^{2}\right)\left[z^{2}-z z_{0}\left(2+q^{2}\right)+z_{0}^{2}+1-q^{2}\right]}, \\
q^{ \pm}=q_{x} \pm i q_{y} \\
z^{ \pm}=z^{x} \pm i z^{y} .
\end{gathered}
$$

Используя соотношения $(22)$, плотность тока $j_{m z}$ можно представить в виде

$$
\begin{aligned}
j_{m z}(\vec{q}, z) \cong & \frac{\mu_{b} c n k_{\mathrm{c}} z q^{2}}{2\left(z+z_{0} q^{2}\right)} \frac{q^{+} z^{-}}{\left[z^{2}-z z_{0}\left(2+q^{2}\right)+z_{0}^{2}+1-q^{2}\right]}- \\
& -\frac{\mu_{b} c n k_{\mathrm{c}} z q^{2}}{2\left(z-z_{0} q^{2}\right)} \frac{q^{-} z^{+}}{\left[z^{2}+z z_{0}\left(2+q^{2}\right)+z_{0}^{2}+1-q^{2}\right]} .
\end{aligned}
$$

Из полученной формулы видно, что величина $z$-компоненты плотности тока намагниченности, связанного со спиновыми колебаниями, в линейном режиме возбуждения спиновых поперечных мод имеет резонансный характер. Отметим, что это свойственно спиновым возбуждениям в высокотемпературных сверхпроводящих системах и является косвенным подтверждением справедливости спин-фононного механизма сверхпроводимости.

Таким образом, в работе показано, что в спектре спиновых возбуждений поперечных спиновых колебаний должны возникать ветви, которые не являются диффузионными в области значений волнового вектора $k<k_{\text {c }}$. Экспериментальные наблюдения таких ветвей колебаний [7] являются косвенным подтверждением сушествования продольной ветви спиновых колебаний в высокотемпературной фазе, которая ответственна за усиление электрон-фононного взаимодействия.

\section{Список литературы}

[1] Н. Н. Боголюбов. Избранные труды в трех томах. Т. 3. Киев: Наукова Думка, 1971 Е.Р. Алабердин, А.А. Вихорев, А.М. Савченко, Б.И. Садовников. ТМФ. 1996. Т. 107. № 1. С. 129; Е. Р. Алабердин, А. А. Вихорев, А. М. Савченко, М. Б. Садовникова. ТМФ. 1999. Т. 120. № 1. С. 144; Е. Р. Алабердин, А. М. Савченко, М. Б. Садовникова. Вестн. МГУ. Сер. физика, астрономия. 1999. № 6. С. 32.

[2] М.А. Савченко, А.В. Стефанович. Флуктуационная сверхпроводимость магнитных систем. М.: Наука, 1986.

[3] B. I. Sadovnikov, A. M. Savchenko. Physica A. 1999. V. 271. P. 411.

[4] M.A. Savchenko, A.V. Stefanovich. Fluctuation superconductivity of Magnetic Systems. Berlin: Springer, 1990.

[5] Ч. Киттель. Квантовая теория твердых тел. М.: Наука, 1967.

[6] Л. В. Жуковская, А.М. Савченко, Б. И. Садовников. Вестн. МГУ. Сер. физика, астрономия. 2002. № 2. С. 71 .

[7] J. Brinckmann, P. A. Lee. Phys. Rev. Lett. 1999. V. 82. № 14. P. 2915.

Поступила в редакцию 1.III.2002 г., после доработки 17.III.2003 г. 